\title{
Design of Fractional-order PID Controllers for Time Delay Systems using Differential Evolution Algorithm
}

\author{
Mohammad Esmaeilzade Shahri ${ }^{1 *}$, Saeed Balochian ${ }^{1}$, Hossein Balochian ${ }^{2}$ and Yudong Zhang ${ }^{3}$ \\ 'Department of Electrical Engineering, Gonabad Branch, Islamic Azad University, Gonabad, Iran; \\ m65esmaeilzade@yahoo.com, saeed.balochian@gmail.com \\ 2Department of Computer Engineering, Ilam Branch, Islamic Azad University, Ilam, Iran; \\ hossein.balochian@gmail.com \\ ${ }^{3}$ School of Computer Science and Technology, Nanjing Normal University, Jiangsu, \\ China, 210023; zhangyudongnuaa@gmail.com
}

\begin{abstract}
This paper aims to club on the scheme of Fractional-Order Proportional-Integral-Derivative (FOPID) controllers for time delay systems. The way is based on a composition of the DE algorithm and the Smith predictor control method to arrive meliorated control efficiency of the time delay process. Lately, Differential Evolution (DE) has been transpired as an ordinary but very powerful materiel for real parameter optimization.

Parameters of FOPID controllers consisted proportionality constant, integral constant, derivative constant, derivative order and integral order, thus its scheme is more intricate than that of conventional integer-order Proportional-Integral-Derivative (PID) controller. Manufacturing of the controller portrayed here is based on user- specified peak overshoot and settling time and has been formulated as a single objective optimization issue. Finally, better simulation results and control performance of the Fractional-Order PID (FOPID) will be showed in these controllers in collation with those of the integer-order PID controllers.
\end{abstract}

Keywords: Differential Evolution, Fractional Order $\mathrm{PT}^{\lambda} \mathrm{D}^{\mu}$ Controller, Smith Predictor Control, Time Delay System

\section{Introduction}

In the past decades, modern control theories have made great advances. Control techniques including optimal control, $\mathrm{H}_{\mu_{1}} / \mathrm{H}_{2}$ control, fuzzy control, neural network control, predictive control, and so on, have been developed. Nevertheless, the Proportional-Integral-Derivative (PID) control technique has still been widely utilized in many industrial applications such as process control, motor drives, flight control, etc. Now a day, more than $90 \%$ control loop in industry are PID control. This is mainly due to the fact that PID controller possesses robust performance to meet the global change of industry process, simple structure to be easily understood by engineers and easiness to design and implement.
The tuning method of PID controllers have been studied intensively in the past such as the well-known Ziegler-Nichols tuning rule ${ }^{1}$ for the first order plus timedelay transfer functions, which models a wide class of processes possessing an S-shape reaction curve in step responses. Another research line for the design of PID controllers is to determine the stabilizing parameter set of PID controller, this set was first shown $\mathrm{in}^{2}$ as convex polygons for delay- free systems by an extension of Hermite-Biehler theorem presented by Pontryagin ${ }^{3}$. Then, the approach was applied to first-order plus time-delay systems and the convex polygon property was extended to this case $\mathrm{e}^{4}$. By using the Nyquist stability criterion, the same results as those in ${ }^{4}$ were obtained which gave an alternative simple derivation ${ }^{5}$. The technique employed in $^{4}$

*Author for correspondence 
was also generalized to the second-order-integrating processes with time-delay ${ }^{6}$. Smith Prediction Control may be the most popular plan for time delay compensation $^{7}$. In the Smith predictor state, the control signal is computed on the foundation of the predicted system conditions and current prediction error. Thus the controller can be designed considering to the delay free section of the system. Some other methods with the alike Smith structure like GPC (Generalized Predictive Control) have been successfully used in PID controller design ${ }^{7,8}$ for delayed SISO systems.

Recently, there are increasing interest to enhance the performance of PID controller by using the concept of fractional calculus, where the orders of derivative and integrals are non-integer. Fractional calculus in non-local makes it able to emphasize mathematically the longmemory. Fractional Order PID controller (FOPID or $\mathrm{PT}^{\mathrm{h}} \mathrm{D}^{\mu}$, where $\lambda$ and $\mu$ are the integrating and derivative orders and they are non-integers) proposed by ${ }^{9}$ is a generalization of the PID controller using fractional calculus. A FOPID controller is characterize by five parameters, i.e., the proportional gain, the integrating gain, the derivative gain, the integrating order and the derivative order. Over the last years, FOPID controllers find many applications in irrigation canal control ${ }^{10}$, temperature tracking ${ }^{11}$, motion control of DC motor ${ }^{12,13}$, boost converter control ${ }^{14}$.

The above research results show that FOPID controller has better performance and robustness than conventional PID controller.

In the literature, many approaches have been proposed to design FOPID controller. These approaches can be classified into two classes: analytic methods and heuristic methods. In the analytic context, the parameters of FOPID controller are tuned by minimizing a nonlinear objective function depending on the specifications imposed by the designers. In ${ }^{15}$, tuning of FOPID controller is recasted as a Quantitative Feedback Theory (QFT) loop shaping problem, where the optimization objective is the high frequency gain of the nominal loop subjected restrictions given by the specifications. ${ }^{16}$ proposed a new analytic method to design FOPID controller by expanding the control loop signal and reference model input and output over a piecewise orthogonal functions. As far as the heuristic methods, rule-based methods and evolutionary algorithm based methods were explored by several authors. $\mathrm{In}^{17}$, a complex of tuning cods was stated based on a first order plus time model of the process by minimizing the integrated absolute error with a border for the maximum sensitivity. Ziegler-Nichols like tuning rules for FOPID controller was given in $^{18}$. Evolutionary algorithms including Genetic Algorithm (GA), Particle Swarm Optimization (PSO) and Electromagnetism like algorithm (EM) are also used to design FOPID controller. Genetic algorithms were adopted by Cao and Meng and $\mathrm{Xue}^{19}$ to design FOPID controller by recasting the problem to an optimization problem. $\mathrm{In}^{20}$, particle swarm optimization was used to design FOPID controller for an AVR system. An Improved Electromagnetism- like Algorithm with Genetic Algorithm (IEMGA) technique was proposed in $^{21}$ for FOPID controller design through minimizing the Integrated-Square-Error (ISE).

Lately, Differential Evolution (DE $)^{22}$ as a plain and impressive plot for global optimization over steady spaces is becoming increasingly popular. In the present work, scheme of FOPID controller using DE algorithm and Smith predictor control styles has been investigated for time delay systems.

The remains of the paper is organized as follows: Section 2 briefs the axioms of fractional calculus needed for presentation of fractional systems. Section 3 provides a brief overview of the algorithms of DE clan and explains a recent state-of-the ort issue of $\mathrm{DE}$ called $\mathrm{DE} / \mathrm{rand}$ / either-or, which were used, in this special task. Section 4 describes how the DE can be applied to the $\mathrm{PT}^{\mathrm{k}} \mathrm{D}^{\mu}$ controller scheme issue when formulated as an optimization task. Section 5 describes the Smith predictive control. The design procedure will be described in section 6 . Results obtained from simulation and observations are given in section 7 and finally, conclusion remarks will be expressed in section 8 .

\section{Essence of Fractional Calculus and Fractional Linear Systems}

The fractional calculus is a 300 years old mathematical discipline, but there are still various mathematical praises that may lead to various conclusions. In fact a unique praise for fractional integration exists. However infinity of formulae could be derived for fractional differentiation. This prevent establishment of systematic theory for fractional linear systems. In other to eschew this obscurity, which is usually relevant to primary status, generally limitation of them to null value is made. 


\subsection{Fractional Integration}

The fractional integral (Riemann-Liouville integral) of a function $f(t)$ is defined by ${ }^{23}$ :

$$
\left(I_{a}^{\pi} f\right)(t) \stackrel{s}{=} \frac{1}{\Gamma(\alpha)} \int_{a}^{t} \frac{f(t)}{(t-t)^{1-x}} d t
$$

where $t>\alpha$ and $\alpha$ is the real positive integration order, $\Gamma(\alpha)$ is the Euler

Gamma function:

$$
\Gamma(\alpha)=\int_{0}^{\infty} e^{-x} x^{\alpha-1} d x
$$

\subsection{Fractional Differentiation}

The Riemann-Liouville definition ${ }^{24}$ is given by:

$$
\begin{aligned}
d^{k L} D_{t}^{\alpha} f(t)= & \frac{1}{\Gamma(m-\alpha)}\left(\frac{d t}{d t}\right) \\
& \int_{a}^{t} \frac{f(t)}{\left(t-t^{t}\right)^{-(t+-a)}} d t
\end{aligned}
$$

where $\mathrm{m}$ is the integer satisfying $m-1<\alpha<m$. It is main to regard that these two compliments are specific occasions of a limitless probability of successive differ integration ${ }^{23}$.

\subsection{Fractional Linear Models}

The Laplace transform of the $f(\mathrm{t})$ integral is imparted $\mathrm{as}^{24}$.

$$
L\left\{I_{0}^{\pi} f(t)\right\}=\frac{1}{s^{\pi}} f(s)
$$

Also, when $f(0)=\Sigma_{0}^{1} f(0)=\ldots=D_{0}^{\alpha} f(0)=0$, the Laplace Transform of the generalized derivative may be imparted as equation 5 :

$$
\left.\left.L^{\mathrm{KL}} D_{0}^{(\pi} f(t)\right\}=L^{c} D_{0}^{(\pi} f(t)\right)=s^{(\pi} f(s)
$$

A Signal Input Signal Output (SISO) Linear Time Constant (LTI) system, relaxed at $t=0$, may be characterized by a differential equation as follows:

$$
\sum_{i=0}^{n} a_{i} D_{0}^{\alpha} y(t)=\sum_{j=0}^{n} b_{j} D_{0}^{j_{i}} t_{i}(t)
$$

Hence, system (6) could be illustrated by the transfer function in the Laplace domain:

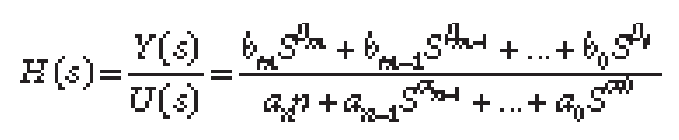

where, $\sigma_{\mathrm{r}}$ and $\boldsymbol{\beta}_{\boldsymbol{c}}$ are ideal real positive, $\boldsymbol{u}(\boldsymbol{t})$ and $\boldsymbol{\gamma}^{(t)}$ are input and output of the system, respectively.

\section{The DE Algorithm and its Modification}

DE inception with NP D-dimensional parameter vectors alike to any other evolutionary algorithm, representing the possible solutions of the problem (Here NP is the number of population and $\mathrm{D}$ the number of parameter defining the problem dimension). We allocate further generations in DE by $G=0,1, \ldots, G_{m}$. Since it is likely that the parameter vectors be uncertain over various generations, we entertain the following impression for representing the ith vector of the population in the current generation as

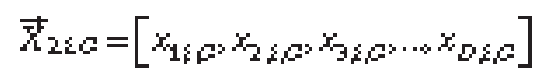

The beginning population (at $G=0$ ) should cover the whole search space as much as possible by uniformly randomizing individuals into the search space limited by the assigned minimum and maximum boundaries:

$$
\begin{aligned}
& Z_{\text {min }}=\left\{x_{1 \text { min }}, x_{2 \text { man }}, \ldots, x_{D \text { min }}\right\} \text { and } \\
& Z_{\text {max }}=\left\{x_{1 \text { max }}, x_{2 \text { max }}, \ldots, x_{D \text { max }}\right\}
\end{aligned}
$$

Hence, we may initialize the $\mathrm{j}^{\text {th }}$ part of the $\mathrm{i}^{\text {th }}$ vector as

$$
x_{j / 0}=x_{j \min }+\operatorname{ran} d_{j}(0,1)\left(x_{j \mathrm{max}}-x_{j \text { min }}\right)
$$

where, rand $(0,1)$ is the $j^{\text {th }}$ polling of a uniformly distributed casual number lying into $(0,1)$ distance. The complete pseudo- code has been described below:

Pseudo-code for the DE algorithm family

\section{Step 1:}

Set the generation number $G=0$, and randomly initialize a population of NP individuals $\mathbb{P}_{\theta}=\left\{\bar{X}_{1 \rho ;}, \bar{X}_{2, \sigma}\right\}$

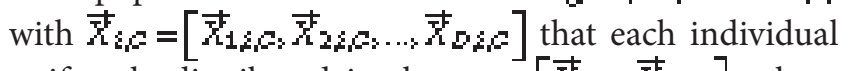
uniformly distributed in the span $\left[\vec{X}_{\min }, \vec{X}_{m \approx x}\right]$, where $\overline{\mathrm{x}}_{\min }=\left\{\mathrm{x}_{1, \text { min }}, \mathrm{x}_{2, \text { min }}, \ldots, \mathrm{x}_{\mathrm{D} \text {,min }}\right.$ and
$\overline{\mathrm{X}}_{\mathrm{m} \boldsymbol{}}=\left\{\mathrm{x}_{1, \text { max }}, \mathrm{x}_{2, \mathrm{~m} \approx}, \ldots, \mathrm{x}_{\mathrm{D} \text { max }}\right\}$ with $i=[1,2, \ldots N \mathrm{~N}]$.

\section{Step 2:}

WHILE cease scale is not contented

DO

FOR $\mathrm{i}=1$ to $\mathrm{NP} / /$ do for each individual sequentially 


\section{Step 2.1: Mutation Step}

Generate a donor vector $\vec{\nabla}_{i}, l=\left\{v_{\varepsilon, \beta}^{1}, \cdots, \nu_{\varepsilon, \rho}^{D}\right\}$ corresponding to the $\mathrm{i}^{\text {th }}$ target vector $\bar{X}_{\mathrm{i}} f$ via one of the different mutation schemes of DE are listed below:

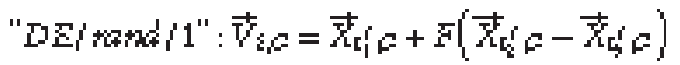

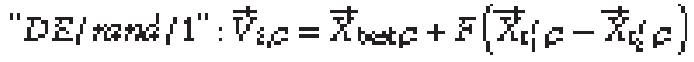

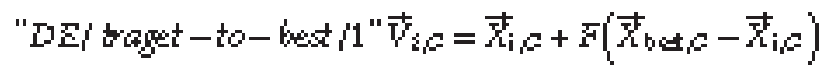

$$
\begin{aligned}
& +F\left(\vec{X}_{t}\left[\rho-\vec{X}_{\mathrm{L}} \rho\right)\right. \\
& \text { "DE/ het } / 2 ": \vec{V}_{b c}=\vec{X}_{b e t} \rho+F\left(\vec{X}_{\mathrm{r}} \rho-\vec{X}_{b_{b}} c\right) \\
& +F\left(\bar{X}_{b} \rho-\vec{X}_{b} a\right)
\end{aligned}
$$

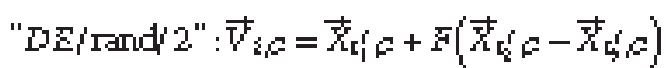

$$
\begin{aligned}
& +F\left(\bar{X}_{b, c}-\bar{X}_{b, c}\right)
\end{aligned}
$$

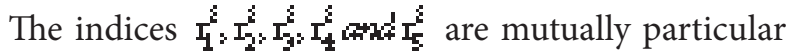
integers selective randomly from the range ${ }^{26}$, NP, which are also different from the index i. These indices are randomly begotten once for each mutant vector. The scaling factor $\mathrm{F}$ is a positive control parameter for scaling the different vectors. $\dot{\mathrm{x}}_{\mathrm{t} \in \mathrm{g}}$ is the best individual vector with the best adaptability function value in the population of generation $\mathrm{G}$.

\section{Step 2.2: Crossover step}

Create a test vector $U_{i \sigma}=\left\{v_{i \rho}^{1}, \ldots, u_{i, \rho}^{D}\right\}$ for the $i^{\text {th }}$ goal vector $\vec{x}_{\mathrm{i} f}$ by binomial crossover (Equation 10 ) or by exponential crossover equation stated following:

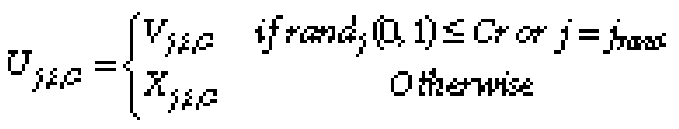

where, and $[0,1) \in[0,1]$ is $j^{\text {th }}$ evaluation for a uniform chance number generator. $j_{\text {rnd }} \in[1,2, \ldots, D]$ is a randomly chosen index, which ensures that $\bar{J}_{\mathrm{i}}, g$ gets at least one part from $\vec{V}_{\mathrm{i}} \Leftrightarrow$.

Or by the arithmetic crossover as follows:

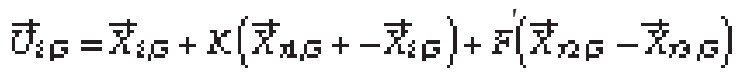

where, $\mathrm{K}$ is the combination ratio, which has been found $\mathrm{d}^{27}$ to be impressive when it is selective as a uniform random repartition from $[0,1]$ and $\mathbf{F}^{\prime}=\mathrm{KF}$ is a new constant here.

\section{Step 2.3: Selection Step}

If we demand the population size to be constant over further generations, the next step of the algorithm will be called for selection to assign whether the goal or the test vector survives to the next generations, i.e. at $G=G+1$. The selection may be outlined as follows:

$$
\vec{X}_{\xi} G+1=\left\{\begin{array}{l}
\vec{U}_{\xi G} \text { if } f\left(\vec{U}_{\xi G}\right) \leq f\left(\bar{x}_{\xi G}\right) \\
\bar{x}_{\xi} G \text { if } f\left(\bar{U}_{\xi} G\right) \leq f\left(\bar{x}_{\xi G}\right)
\end{array}\right.
$$

where, $f(\bar{x})$ is the function should be minimized.

Assign the test vector $\vec{\nabla}_{i} G$

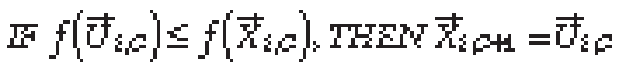

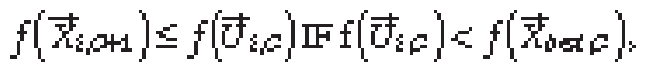

$$
\begin{aligned}
& \text { THEN } \bar{X}_{\text {det }} c=\bar{U}_{\varepsilon} \rho
\end{aligned}
$$

In the basic DE mutation scheme, a constant factor "F" is used to scale the difference vector $\left[\vec{X}_{s}(t)-\vec{X}_{j}(t)\right]$. The common selection for this control parameter is a number between and 0.4 and 1 . We offer to vary this scale factor randomly in the range $(0.5,1)$ using the relationship

$$
\bar{F}=0.5\left(1+r_{a} \text { and }(0,1)\right)
$$

where, rand $(0,1)$ is a uniformly distributed random number within the range $[0,1]$. The average value of the scale factor is 0.75 . This permits for stochastic alteration in the amplification of the difference vector and thus supports to keep population diversity as the search progresses. $\mathrm{In}^{28}$, it has already been shown that the DERANDSE (DE with random scale Factor) can meet or beat the classical DE and also some versions of PSO in a statistically significant style. Ashore this, here we also decrease the crossover $\mathrm{CR}$ rate linearly with time from $C R_{\text {max }}=0.1$ to $C R_{\text {min }}=0.5$. If $C R=1$, whole components of the parent vector are sub by the difference vector operator matching to ${ }^{15}$. But at the later stations of the optimization process, if $C R$ be decreased, then more components of the parent vector are inherited by the offspring. Such tuning of $C R$ support to prospect the search space exhaustively at the beginning, but adjust the movements of test solutions finely during the later stages of the search, so that they can prospect the interior of a relatively small space in which the suspected global optimum lies. The time variation of $C R$ may be imparts in the sorrow of the following equation:

$$
C R=\left(C R_{\max }-C R_{\min }\right)\left(\frac{G_{\operatorname{mox}}-G}{G_{\max }}\right)+C R_{\min }
$$


where, $C R_{\mathrm{max}}$ and $C R_{\min }$ are the maximum and minimum values of crossover rate $C R, G$ is the current generation number and $G_{m=\infty}$ is the maximum number of permissive generations. Then performing a series of test, we meet that the $\mathrm{DE} / \mathrm{rand} / \mathrm{l} /$ bin scheme (Equation 10) armed with these corrections can outperform all other classical $\mathrm{DE}$ variants for the checked controller design matter in this article.

\section{The DE-based Scheme of Fractional $\mathrm{PI}^{\lambda} \mathrm{D}^{\mu}$ Controllers}

\subsection{The FOPID Controller}

A PID controller is a common control loop feedback mechanism extensively applied in industrial systems. The following transfer function explained to an integer- order PID controller:

$$
G_{q}(s)=k_{y}+k_{s} s^{-1}+k_{d a} s
$$

The PID controller computing (algorithm) include three apart parameters: the proportional $\left(k_{b}\right)$, the integral $\left(k_{2}\right)$ and derivative $\left(k_{\alpha}\right)$ time constants. The proportional portion defines the response to the current error, the integral defines the response on the basis of the sum of latter errors and the derivative defines the response to the rate at which the error has been changed. The weighted sum of these three acts is handled to adjust the process by a control detail such as the location of a control value or the power supply of a heating element. Figure 1 signs the block diagram of a common closedloop-control system involving the PID controller. PID controllers belong to main industrial controller and hence are targets of steady fuss to amend their quality and robustness. It is feasible to use fractional-order controllers with non-integer derivation and integration portions to amend PID controllers.

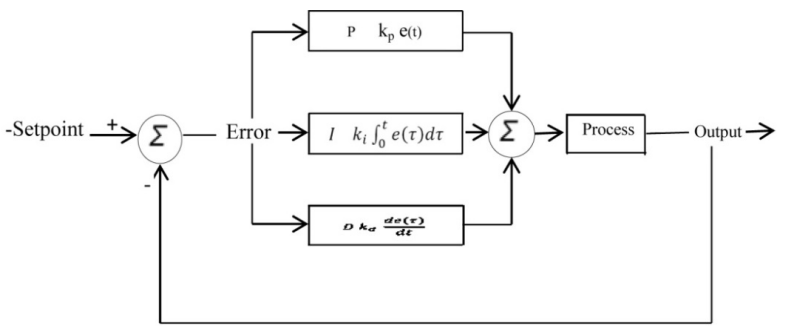

Figure 1. A generic closed-loop process control system with PID controller.
Sequent the works of ${ }^{9}$, we may goal to generalize PID controller, of which can be called the $\mathrm{PT}^{\mathrm{h}} \mathrm{D}^{\mu}$ controllers because of involving an integrator with order of $\lambda$ and a differentiator with order of $\mu$. The continuous transfer function of such a controller is same to:

$$
G_{e}(s)=k_{b}+k_{s} s^{-\lambda}+k_{\alpha} s^{\prime \prime}\left(\lambda_{s} \mu>0\right)
$$

The output response of the $\mathrm{PT}^{\mathrm{h}} \mathrm{D}^{\mu}$ controllers in time period may be explained as:

$$
U(t)=k_{g} e(t)+k_{c} D^{-\lambda} e(t)+k_{a x} D^{\nu} e(t)
$$

where, $\lambda=+1, \mu=+1$ implies normal PID controller, for $\lambda=0, \mu=+1$, we achieve a normal PD controller, $\lambda=+1, \mu=0$ imparts normal PI controller and $\lambda=0, \mu=0$ imparts a specific instances of the fractional $\mathrm{PT}^{\mathrm{h}} \mathrm{D}^{\mu}$ controller. As can be understood from Figure 2, the FOPID controller can generalize the integer-order PID controller and can develop it from point to plane. This development reason that controller design is more flexible and we can control our real-world processes more literally.

\subsection{Formulation of the Objective Function}

The procedure for scheme stated here is based on the root locus method (dominant roots method) of structure integral PID controllers ${ }^{29}$. Alike to traditional root locus method based on the user characteristics of peak overshoot MP and settling time, we find out the damping ratio $\xi$ and the un-damped natural frequency $\omega_{0}$ of the closed-loop system should be schemed, thus dominant poles will be

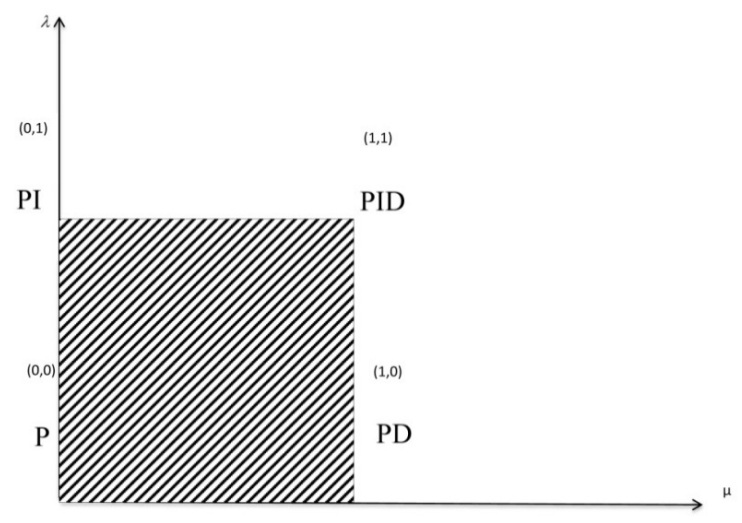

Figure 2. Generalization of the FOPID controller: from point to plane. 


$$
I_{1,2}=-\zeta \omega_{0} \pm j \omega_{0} \sqrt{1-\zeta^{2}}=-x \pm j y
$$

Let the closed-loop transfer function be as follows:

$$
\frac{C(s)}{R(s)}=\frac{G(s)}{1+G(s) H(s)}
$$

where, the transfer function of the process is $G_{p}(s)$ to be controlled and that of the controller is $\mathrm{G}_{\mathrm{C}}(\mathrm{s})=\mathrm{U}(\mathrm{s}) /$ $\mathrm{E}(\mathrm{s})$ and $\mathrm{G}(\mathrm{s})=\mathrm{G}_{\mathrm{C}}(\mathrm{s}) \mathrm{G}_{\mathrm{p}}(\mathrm{s})$. We suppose unity feedback portion, i.e., $\mathrm{H}(\mathrm{s})=1$ from Equation 25 the characteristics of the equation of the closed-loop system are:

$$
1+G(s) H(s)=0 \Rightarrow 1+G_{g}(s) \cdot G_{c}(s) \cdot 1=0
$$

Now the dominant poles of the system are the zeros points of this characteristic equation, so they will clearly content the equation. Thus from (26) we get:

$$
\begin{array}{r}
1+\left[k_{b}+k_{i}(-x+j y)^{-\lambda}+k_{b}(-x+j y)^{\mu}\right] \\
G_{b}(-x+j y)=0
\end{array}
$$

This equation has whole five passive parameters involving $k_{b}, k_{2}, k_{a}, \lambda$ and $\mu$. Let $R$ be the real portion of the abstruse expression (27), I be the imaginary portion of the abstruse expression (27) and $\Psi$ the phase angle $=$ $\tan ^{-1}(\mathrm{I} / \mathrm{R})$.

Now we can describe objective function as follows:

$$
T\left(k_{\dot{b}}, k_{i}, k_{b}, \lambda_{0}, a\right)=|I|^{2}+|\not|^{2}+|\psi|^{2}
$$

Our target is to find an optimal solution set $\left\{k_{\dot{p}}, k_{\varepsilon}, k_{\alpha}, \lambda_{s}, \mu\right\}$ in which $J=0$. Here, function said above has been minimized with modified DE/rand/1/bin algorithm.

\subsection{Vector Representation in DE}

The solution space of Equation 28 is five-dimensional, the five dimensions are $\left\{k_{\dot{b}}, k_{3}, k_{\alpha}, \lambda_{,}, \mu\right\}$. So each parameter vector has five components in $\mathrm{DE}$, i.e., the $\mathrm{j}^{\text {th }}$ population member at $\mathrm{G}^{\text {th }}$ generation may be given as follows:

$$
\bar{x}_{i, a}=\left(k_{b}, k_{z}, k_{b}, \lambda_{3}, \mu\right)^{\tau}
$$

We given the appendix numerical ranges for each parameter based on applied regard of the PID controller scheme ${ }^{29}$ :

$$
\begin{aligned}
& 1 \leq k_{p} \leq 1000 \\
& 0 \leq \lambda_{,}, u \leq 2 \\
& 1 \leq k_{z}, k_{b} \leq 900
\end{aligned}
$$

\section{Overview of Smith Predictive Control}

The block diagram of the convention Smith predictive control is shown in Figure 3. $G_{C}(s)$ denotes a rational function characterizing the compensator called primary controller. $G_{p}(s)$ denotes a stable, strictly proper rational function characterizing the delay-free part of the plant. ' explain a positive constant standing for the time-delay.

$\mathrm{G}_{\mathrm{p}}(\mathrm{s})$ and $\mathrm{s}$ are obtained through modeling process. The inner loop works to eliminate the actual delayed output as well as to get the feedback of the predicted output to the primary controller. This makes it possible to design the primary controller assuming no time-delay in the control loop.

From Figure 3, the transfer function of the closed loop system after compensation is:

$$
\phi(s)=\frac{G_{c}(s) G_{g}(s)}{1+G_{c}(s) G_{g}(s)} e^{-z s}
$$

The expression Equation 31 is equivalent to the block diagram shown in Figure 4.

This would move the time delay outside the control loop. Since there would be no delay in the feedback signal, the response of the system would be in proved. That is, the system can eliminate the influence of time- delay on system $^{30}$.

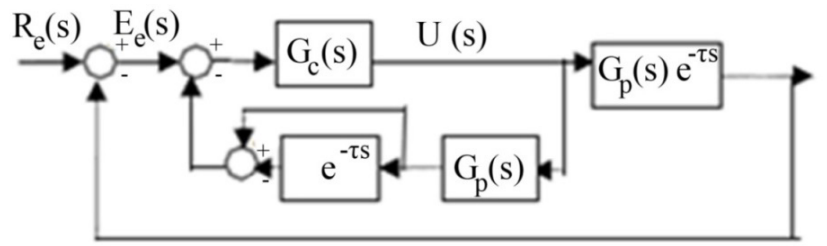

Figure 3. Block diagram of the conventional Smith predictive control.

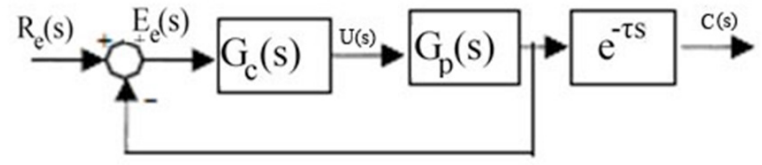

Figure 4. Equivalent block diagram of the conventional Smith predictive control. 


\section{Design Procedure}

A proposed design method is based in the DE algorithm and the Smith predictor control method. It includes the under original steps:

\section{Step 1:}

Consider the plant with time delay $G(s)=G_{g}(s) e^{-z s}$

\section{Step 2:}

Calculation of the optimal FOPID controller parameters for the process $\mathrm{G}_{\mathrm{p}}(\mathrm{s})$ using $\mathrm{DE}$ algorithm (where assumed $\mathrm{M}_{\mathrm{p}}$ and $\mathrm{T}_{\mathrm{S}}$ to be known).

\section{Step 3:}

Identification of transfer function $\mathrm{G}_{\mathrm{C}}(\mathrm{s})$ using Equation 22.

\section{Step 4:}

Put on $\mathrm{G}_{\mathrm{C}}(\mathrm{s})$ in Figure 4 .

\section{Simulation Results}

We have examined our proposed manner on two own instances for scheme quandary. All the scheme instances follow the basic framework comprehensive in portion. The first problem includes the flotation column, in which it is favorable for us that it has a settling time of 10 s and overshoot of $10 \%$. The second problem also includes an oscillatory system, which should be controlled for obtaining a peak overshoot of $30 \%$ and settling time of 30s in the closed-loop response. The parameters of $\mathrm{DE}$ algorithm are selective as follows:

Pop size: 100

CR max: 1.0

DR min: 0.5

Scale factor F: Uniformly distributed random number between 0.5 and 1.0 with mean value 0.75

\section{Example 1: Flotation Column}

The actual production environment provided a transfer function for pulp level in the flotation column. It can be expressed as an inertial first-order system with a pure time delay where the value is regarded as an inertial first-order system $^{31,32}$. The transfer function for pulp level in the flotation column and the value transmission, given in $\operatorname{Ref}^{31}$ and $^{32}$ are:

$$
\begin{aligned}
& G_{1}(s)=\frac{2}{0.4 s+1} \\
& G_{2}(s)=\frac{4}{4 s+1} e^{-2 s}
\end{aligned}
$$

Applying the DE algorithm to the process model

$$
G_{g}(s)=\frac{g}{(0.4 s+1)(4 s+1)}
$$

We get the optimized parameters for the fractional order PID controller:

$$
k_{y}=1, k_{i}=1.0437, k_{i c}=1.4657, \lambda=1.1706, \mu=0.4117
$$

The transfer function for the fractional order PID controller is:

$$
G_{\varepsilon}(s)=1+\frac{1.0437}{s^{1506}}+1.4657 s^{0.4117}
$$

If we set $\lambda=1$ and $\mu=1$ before running the $\mathrm{DE}$ algorithm, we obtain the three optimized parameters for the integer order PID controller. The optimized parameters for the integer order PID controller are:

$$
k_{y}=1.4139, k_{i}=2.1256, k_{c}=1.7939
$$

The transfer function for the integer order PID controller is:

$$
G_{c}(s)=1.4139+\frac{2.1256}{s}+1.7939 s
$$

The simulation block diagram for a flotation column with fractional PID controller and integer PID controller is shown in Figure 5. The unit- step responses are show in Figure 6. Table1 lists the performance indices in the time domain of the step responses shown in Figure 6.

\section{Example 2: Oscillatory System}

Consider an oscillatory system with open-loop transfer function $^{25}$.

$$
G(s)=\frac{1}{\left(s^{2}+s+1\right)(s+2)^{2}} e^{-01 s}
$$

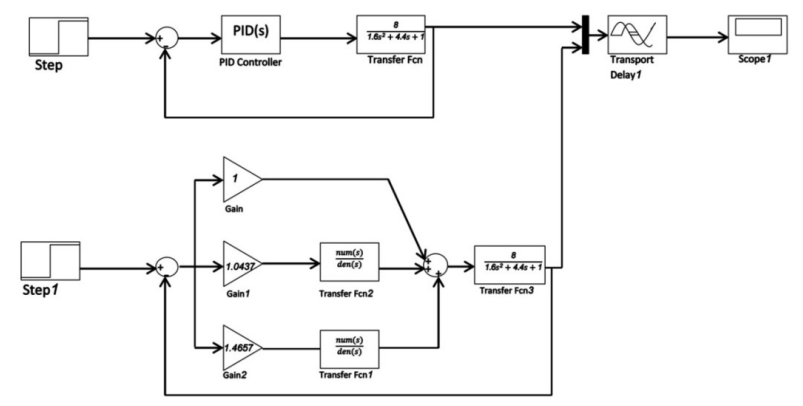

Figure 5. Simulation block diagram for flotation column with fractional PID controller and integer PID controller. 


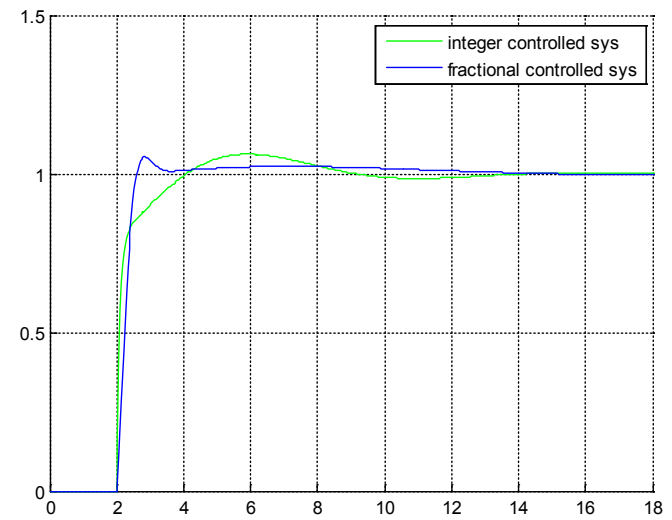

Figure 6. Unit-step responses of the flotation column with Fractional PID controller and Integer PID controller.

Table 1. Comparison

\begin{tabular}{lllll}
\hline $\begin{array}{l}\text { Type of } \\
\text { controller }\end{array}$ & $\begin{array}{l}\text { Peak } \\
\text { time } \\
(\mathrm{sec})\end{array}$ & $\begin{array}{l}\text { Peak } \\
\text { overshoot }(\%)\end{array}$ & $\begin{array}{l}\text { Rise } \\
\text { time } \\
(\mathrm{sec})\end{array}$ & $\begin{array}{l}\text { Settling } \\
\text { time } \\
(\mathrm{sec})\end{array}$ \\
\hline Fractional PID & 2.7 & 8.27 & 2.375 & 4.24 \\
Integer PID & 4.26 & 9.4 & 2.643 & 9.09 \\
\hline
\end{tabular}

Applying the de algorithm to the process model

$$
G_{\theta}(s)=\frac{1}{\left(s^{2}+s+1\right)(s+2)^{2}}
$$

We get the optimized parameters for the fractional PID controller is

$$
k_{y}=1.1427, k_{i}=19163, k_{\alpha x}=1.0944, \lambda=1.3227, \mu=0.4986
$$

The transfer function for the fractional PID controller is

$$
G_{c}(s)=1.1427+\frac{1.9163}{s^{13 W}}+1.0044 s^{0.4 * x}
$$

With set $\lambda=1$ and $\mu=1$ we obtain three optimized parameters for the integer PID controller as: $k_{y}=1, k_{z}=2.0537, k_{\alpha x}=9.4776$. The transfer function for the integer PID controller is: $G_{j}(s)=1+\frac{2.0537}{s}+9.4776 s$ The simulation block diagram for oscillatory system with fractional PID controller and integer PID controller is shown Figure 7. The unit-step responses are shown in Figure 8. A comparison of time domain specifications tabulated as given in Table 2 .

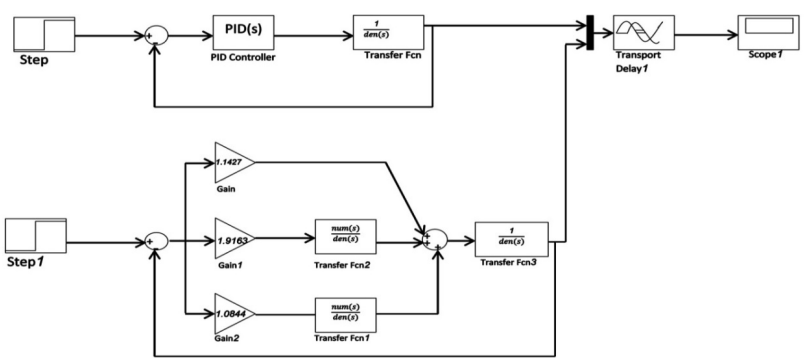

Figure 7. Simulation block diagram for oscillatory system with fractional PID controller and integer PID controller.

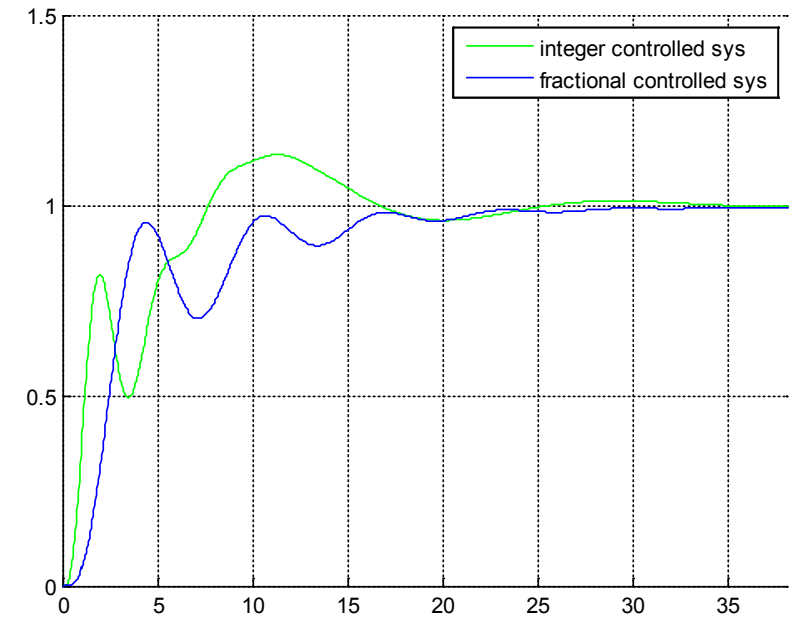

Figure 8. Unit-step responses of the oscillatory system with Fractional PID controller and integer PID controller.

Table 2. Comparison

\begin{tabular}{lllll}
\hline $\begin{array}{l}\text { Type of } \\
\text { controller }\end{array}$ & $\begin{array}{l}\text { Peak } \\
\text { time } \\
(\mathrm{sec})\end{array}$ & $\begin{array}{l}\text { Peak } \\
\text { overshoot }(\%)\end{array}$ & $\begin{array}{l}\text { Rise } \\
\text { time } \\
(\mathrm{sec})\end{array}$ & $\begin{array}{l}\text { Settling } \\
\text { time } \\
(\mathrm{sec})\end{array}$ \\
\hline $\begin{array}{l}\text { Fractional } \\
\text { PID }\end{array}$ & - & 0 & 2.53 & 21.6 \\
Integer PID & 11.3 & 13.4 & 6.24 & 23.4 \\
\hline
\end{tabular}

\section{Discussion and Conclusion}

We proposed a sagacious optimization method based on the DE algorithm for modeling FOPID controllers and smith predictor procedure for time delay systems in this article. Fractional calculus can purvey original and higher efficiency extension for FOPID controllers. Although, the difficulties of scheming FOPID controllers addition, because FOPID controllers also take into account the derivative order and integral order in comparison with 
common PID controllers. Using fractional PID controller we have significantly reduce percentage overshoot and rise time and settling time. Simulation results show that the fractional PID controller has superior performance than integer PID controller.

\section{References}

1. Ziegler JG, Nichols NB. Optimum setting for automatic controller. Transactions ASME 64. 1942.

2. Ho M-T, Data A, Bhattacharyya SP. Design of P, PI and PID controller for interval plants. Proc. American control conference; 1988; Philadelphia, PA, USA.

3. Pontryagin LS. One the zeros of some elementary transcendental function. American Mathematical Society Translation. 2. 1955.

4. Silva GJ. Data A, Bhattacharyya SP. New results on the synthesis of PID controllers. IEEE Trans Automat Contr. 2002; 47(2):c241-52.

5. Marteli G. Comment on new results on the synthesis of PID controller. IEEE Trans Automat Contr. 2005; 50(9):1468-9.

6. Ou L-L, Zhang WD, Gu D. Sets of stabilizing PID controllers for second-order integrating process with time- delay. IEEE Proc Contr Theor Appl. 2006; 153(5):607-14.

7. Tan KK, Lee TH, Leu FM. Optimal Smith predictor design based on a GPC approach. Ind Eng Chem Res. 2002; 41:1242-48.

8. Tan KK, Hung SN, Lee TH. Development of a GPC-based PID controller design for unstable systems with dead time. ISA Trans. 2000; 39(1):55-70.

9. Podlubny I. Fractional-order systems and $\mathrm{PI}^{\lambda} \mathrm{D}^{\mu}$ controllers. IEEE Trans Automat Contr. 1999; 44(1):208-14.

10. Domingues J, Valerio D, Da Costa JS. Rule-based fractional control of an irrigation canal. Proceedings 35th Annual Conference of IEEE Industrial Electronics IECON 09; 2009 Nov 3-5; Portugal. IEEE; 2009. p. 1712-7.

11. Ahn HS, Bhambhani V, Chen RQ. Fractional-order integral and derivative controller for temperature profile tracking. Sadhana, Indian Academy of Sciences. 2009; 34:833-50.

12. Petras I. Fractional-order feedback control of a Dc motor. J Electr Eng. 2009; 60:117-28.

13. Xue DY, Zhao CN, Chen YQ. Fractional order PID control of a DC motor with elastic shaft: A case study. American Control Conference, 2006; 2006 Jun 14-16; Minneapolis, MN.

14. Tehrani K, Amirahmadi A, Rafiei S, Griva G, Barrandon L, Hamzaoui M, Rasoanrivo L, Sargos F. Design of fractional-order PID controller for boost converter based on multi-objective optimization. 4th International Power Electronic and Motion Control Conference; 2010 Sep 6-8; Ohrid. IEEE; 2010. p. 179-85.

15. Cervera J, Banos A, Monje CA, Vinagre B. Tuning of fractional PID controllers by using QFT. 32nd Annual
Conference on IEEE Industrial Electronics, IECON 2006; 2006 Nov 6-10; Paris. IEEE; 2006. p. 5402-7.

16. Bouafoura MK, Braiek NB. $\mathrm{PI}^{\lambda} \mathrm{D}^{\mu}$ controllers design for integer and fractional plants using piecewise orthogonal functions. Comm Nonlinear Sci Numer Simulat. 2010; 15(5):1267-78.

17. Padula F, Visili A. Tuning rules for optimal PID and fractional-order PID controllers. Journal of Process Control. 2011; 21:69-81.

18. Valerio D, Da Costa JS. Tuning of fractional PID controller with Ziegler-Nichols-type laws. Signal Processing. 2006; 86:2771-84.

19. Xue D, Meng L. Design of optimal fractional-order PID controller using multi-objective GA optimization. Control and Decision Conference (CCDC); 2009 Jun 17-19; Guilin. IEEE; 2009. p. 3849-53.

20. Sadati M, Zamani M, Karimi-Ghartemani N, Parniani M. Design of a fractional order PID controller for on AVR system using particle swarm optimization. Control Engineering Practice. 2009; 17(12):1380-7.

21. Chang FK, Lee CCh. Fractional-order PID controller optimization via improved electromagnetism-like algorithm. Expert Syst Appl. 2010; 37:8871-8.

22. Storn, Lampinen J, Price K. Differential Evolution-A practical approach to global optimization. Springer, New York: 2005.

23. Maricher OI, Samko SG, Kilbas AA. Fractional integrals and derivative: theory and applications. Amsterdam: Gordon and Breach Science Publishers; 1993.

24. Miller KS, Ross B. An Introduction to the Fractional Calculus and Fractional Differential Equations. San Fransisco: Wiley-Intersience Emission. 1993.

25. Qing-Gue W, Tong- Heng L, Ho- Wang F, Qiang B, Yu Z. PID tuning for improved performance. IEEE Trans Contr Syst Tech. 1999;7(4):457-65.

26. Oldham KB, Spanier J. The Fractional Calculus. New York: Academic Press; 1974.

27. Price K. An introduction to differential evolution, New Ideas in optimization. UK; 1999.

28. Chakraborty UK, Das S, Konar A. Two improved differential evolution schemes for faster global search. Proceedings of ACM-SIGEVOCECCO; 2005 Jun 25-29; Washington, DC. p. 991-8.

29. Asrtom K. Haggloud T. PID controller, theory, design and tuning. Research Triangle Park: Instrument Society of American; 1995.

30. Xi AM. Computer control technology. Beijing, China: Higher Education Press, 2004(in Chinese).

31. Liao Q, Xu WL, Chu YF. Study on control algorithm of pulp level for self-suction flotation machine. Metal Mine. 2004; 4:39-5.

32. Chu YF, Xu WL, Liao Q. Decentralized control of cascade flotation cells. Metal Mine 2004; 2:20-3. 